\title{
In Vitro Cytotoxicity and Transplantation Protection by Autologous Natural and Activated Killer Cells Against an In Vitro Transformed Tumorigenic Fibroblast Line
}

\author{
A Case Study \\ Sally A. Wilhelm and Bijay Mukherii \\ Department of Medicine, The University of Connecticut School of Medicine, Farmington, Connecticut 06032
}

\begin{abstract}
Cytotoxic immune response by autologous natural killer (NK) cells against a spontaneous in vitro transformed tumorigenic fibroblast line, VIP-F:T, was studied in a $4 \mathrm{~h}{ }^{51} \mathrm{Cr}$-release microcytotoxicity assay and in a tumor cell neutralization technique in vivo in nude mice. Although highly cytotoxic against the NK prototype target K562, the autologous NK cells in their nascent state were only marginally cytotoxic against VIP-F:T and unreactive against the autologous normal fibroblasts, Pen-F2. Autologous NK activity against VIP-F:T could, however, be induced by 2-16-h treatment of the NK cells with several species of interferon and by interferon-free interleukin 2 (IL-2). In vitro co-culture (IVC) in IL-2 of autologous peripheral blood lymphocytes (PBL) against VIPF: $T$ was shown by fluorescence activated cell sorting and by cold target competition experiments to generate almost exclusively an effector population bearing HNK-1 and Leu-11a phenotypes which exhibited receptor specificity for VIP-F:T distinct from receptors on Pen-F2 or K562 cells. PBL, cocultured in IL-2 against Pen-F2 or K562, or cultured in IL-2 alone, generated high levels of nonspecific killing and showed no receptor specificity. Identical IVC in IL-2 of autologous PBL against a melanoma line, VIP (PBL and the VIP line derived from the same patient from whom the VIP-F:T line was also derived), and similar IVC in IL-2 of several other autologous PBL against their corresponding target cell lines (established from surgical specimens) generated cytotoxic responses involving cytotoxic populations bearing $\mathrm{T} 8$ as well as HNK-1 phenotypes; but the cytotoxic activities in none of these systems showed target receptor specificity. Autologous PBL, co-cultured against VIP-F:T in IL-2, were shown to be capable of rejecting tumorigenic challenge with VIP-F:T.3 (a clone of VIP-F:T) in nude mice at effector to VIP-F:T ratio of 10:1. The protective effect of the co-culture activated PBL was abrogated if the HNK-1 + cells were depleted from the effector population. Our data, thus, demonstrate specificity of cytotoxic reactivity which, by phenotypic markers, can be characterized as HNK-1 and Leu 11a+ cells under these experimental conditions against this particular in vitro transformed VIP-F: $T$ line. In addition, this study shows that similar studies of cytotoxic autologous reactivities against in vitro transformed
\end{abstract}

Address reprint requests to Dr. Mukherii.

Received for publication 7 May 1984 and in revised form 3 December 1984.

J. Clin. Invest.

(C) The American Society for Clinical Investigation, Inc. 0021-9738/85/04/1162/07 \$1.00

Volume 75, April 1985, 1162-1168 target cell lines will provide valuable information on the subject of NK-mediated surveillance against human neoplasia.

\section{Introduction}

The concept of immunosurveillance against cancer (1) endures despite controversies $(2,3)$. Attention has been focused lately on natural killer $(\mathrm{NK})^{1}$ cells (4) as potential custodians of surveillance against neoplasia. Despite considerable research, the relevance of natural killing in surveillance against neoplasia has been difficult to determine due largely to the use of prototype target cell lines or cells derived from overt tumors that presumably developed after escaping immunosurveillance. The role of natural killing in surveillance against cancer would be more ideally approached with autologous targets that were transformed in vitro. Using such an approach, we show here the existence of natural killing in blood lymphocytes, as defined operationally and by phenotypic characteristics, against an in vitro transformed tumorigenic autologous human cell line, VIP-F:T. In this system, cytotoxicity (CTX) against the autologous transformed target is inducible with interferon (IFN), interleukin-2 (IL-2), and by in vitro co-culture (IVC) against the autologous transformed target in the presence of exogenous IL-2. We further show that, while IFN or IL-2 induces nonspecific killing, IVC in IL-2 generates cytotoxic effectors which exhibit NK cell phenotype and express specificity for VIP-F:T target receptors distinct from target receptors on autologous non-transformed fibroblasts and on the NK prototype target $\mathrm{K} 562$. In addition, data is presented on in vivo corroboration of the cytotoxicity of the in vitro sensitized effector cells, which shows their ability to impart transplantation rejection of tumorigenic challenge with the VIP-F:T cells in nude mice.

\section{Methods}

Target cells. The VIP-F:T line, developed in our laboratory as a spontaneous in vitro transformation of cultured normal human skin fibroblasts that grow in an anchorage-independent fashion in soft agar and produce tumors histologically and ultrastructurally consistent with sarcomas in nude mice, has been partially described (5). A detailed characterization (including HLA types) of the VIP-F:T line, VIP-F:T.3 (a clone of VIP-F:T), and another autologous normal fibroblast line, Pen-F2, has been presented elsewhere (6). The Pen-F2 line grows as a contact-inhibited monolayer, shows no anchorage-independent growth,

1. Abbreviations used in this paper: CMC, cell-mediated cytotoxicity; CTL, cytotoxic T lymphocytes; CTX, cytotoxicity; FACS, fluorescence activated cell sorter; IFN, interferon; IL-2, interleukin-2; IVC, in vitro co-culture; LAK, lymphokine activated killing; LGL, large granular lymphocytes; NK, natural killer; rIFN- $\gamma$, recombinant interferon- $\gamma$; rIL-2, recombinant interleukin-2. 
and is not tumorigenic in nude mice. The melanoma line, VIP, derived from the same patient from whom the in vitro transformed fibroblast line VIP-F:T was also derived, has been described $(5,6)$. The melanoma line VIP expresses Ia antigen constitutively, whereas the VIP-F:T line exhibits no Ia antigen. Several other melanoma lines that were established in our laboratory have also been used. Tissue culture was performed in Hams F-10 media (Gibco Laboratories, Grand Island, NY) that was supplemented with $10 \%$ fetal calf serum (Gibco Laboratories).

Interleukin 2. The partially purified IL-2 (Electro-Nucleonics, Silver Spring, MD) used in these experiments had no detectable IFN- $\alpha$ or IFN- $\gamma$ activity (tested in standard IFN bioassay in the Interferon Laboratory, Memorial Sloan-Kettering Cancer Center, New York, NY, courtesy of Mathilde Krim). In addition, interferon-free IL-2 that was purified from conditioned media of the Gibbon leukemia cell line MLA-144 (7) (a gift of Harvey Rabin, National Cancer Institute, Frederick, MD) and recombinant DNA-produced IL-2 (Amgen, Thousand Oaks, CA) were also used in selected experiments.

Effector cells. Peripheral blood mononuclear cells were isolated in Ficoll-Hypaque gradient and depleted of macrophages (referred to henceforth as peripheral blood lymphocytes [PBL]) by 2-h adherence in plastic flasks as described earlier (5). Large granular lymphocytes (LGL), which were recently described as prime mediators of NK activity $(4,8)$, were isolated by standard technique $(8)$ from macrophagedepleted PBL in the interface between the first and second layers of six 2.5-ml gradients of Percoll (Pharmacia Fine Chemicals, Piscataway, $\mathrm{NJ}$ ) ranging from $45 \%$ (R.I. 1.3432) and 57.5\% (R.I. 1.3454).

Effector cell sorting. A fluorescence activated cell sorter (FACS IV; B-D FACS Systems, Sunnyvale, CA) was utilized to obtain effector cell population depleted of cells (negative sorting) or to obtain positively sorted cells bearing HNK-1, Leu-11a, or OKT8 phenotypes (Leu-7 and Leu-11a monoclonal antibodies were obtained from BectonDickinson \& Co., Sunnyvale, CA; and OKT8 monoclonal antibody was obtained from Ortho Pharmaceutical Corp., Raritan, NJ). Staining procedure of the effector cells with respective reagents has been published earlier (5).

Depletion of NK cells by complement-mediated lysis of HNK-1 antibody coated cells. Thoroughly washed, sensitized effector cells were incubated with Leu-7 (HNK-1) antibody $\left(1 \mu \mathrm{g} / 10^{6}\right.$ cells) in ice for 30 $\mathrm{min}$. The cells were washed twice and reincubated with rabbit low tox$\mathrm{H}$ complement (Cedarlane Laboratories, Hornby, Ontario, Canada) at a 1:1 dilution at $36^{\circ} \mathrm{C}$ with frequent agitation. The cells were washed three times, percentage lysis was determined by viability count by trypan blue dye exclusion. In pilot experiments, the percentage of cell lysis closely correlated (95-105\%) with the percentage of Leu-7+ cells determined by immunofluorescence technique in FACS (B-D FACS Systems).

In vitro co-culture. IVC of effector cells was carried out with unfractionated blood mononuclear cells in co-culture against irradiated target cells. Briefly, effector cells $\left(1 \times 10^{6} / \mathrm{ml}\right)$ were co-cultured with irradiated $(4,500 \mathrm{rad})$ autologous or allogeneic targets $\left(1 \times 10^{4} / \mathrm{ml}\right)$ in Hams F-10 medium and IL-2 $\left(5 \times 10^{2} \mathrm{U} / \mathrm{ml}\right)$. Cultures were fed with IL-2 containing medium three times weekly.

Microcytotoxicity $(C M C)$ assay. The $4 \mathrm{~h}{ }^{51} \mathrm{Cr}$-release assay has been described earlier (5).

Cold target competition assay. The standard technique of inhibition of cytotoxicity by cold target interaction has been followed (9). Briefly, CMC assays were set up in the usual manner. Immediately after addition of the effector cells, unlabeled (cold) targets of various derivations were added at different concentrations to a constant number of labeled targets. In each assay, dose-response analysis of percentage inhibition was carried out.

In vivo tumor cell neutralization assay. 36-d-old female CD-1 (nu/ $n u$ ) mice (Charles River Breeding Laboratories, Wilmington, MA) were injected subcutaneously with $0.1 \mathrm{ml}$ inocula with $1 \times 10^{6}$ viable VIPF:T.3 cells, alone or mixed with $1 \times 10^{7}$ VIP-F:T sensitized viable effector cells (Incidence of positive tumor take at $1 \times 10^{6}$ VIP-F:T.3 inocula was determined to be $100 \%$ in earlier pilot experiments.). All animals were carefully inspected for visible and palpable tumor growth regularly. Palpable tumors were verified by histologic examinations.
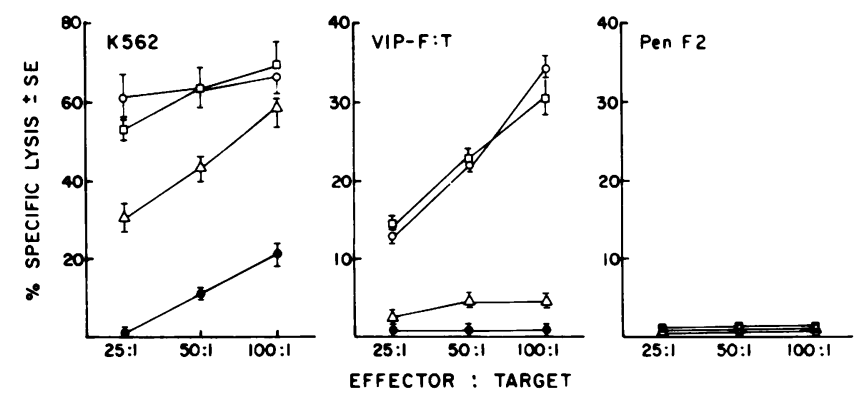

Figure 1. Cytotoxicity by PBL and LGL and the effects of IFN- $\alpha$ and IL-2 on cytotoxicity by the LGL against $\mathrm{K} 562$ and the autologous targets. LGL $\left(5 \times 10^{5} / \mathrm{ml}\right)$ were cultured overnight $(16 \mathrm{~h})$ with the following: $\Delta$, medium alone; $\square, 5 \times 10^{2} \mathrm{U} / \mathrm{ml}$ of IFN- $\alpha$; and $\circ, 5$ $\times 10^{2} \mathrm{U} / \mathrm{ml}$ of partially purified IL-2. $\bullet$, cytotoxicity of the PBL cultured overnight in medium alone.

\section{Results}

Cytotoxicity by PBL and NK cells against autologous targets and K562. Fig. 1 shows the cytotoxic activities of freshly isolated PBL and LGL against the NK prototype target K562 and the autologous targets VIP-F:T and Pen-F2. Also shown in Fig. 1 is the effect of biological response modifiers IFN- $\alpha$ (Meloy Laboratories, Inc., Springfield, VA) and IL-2 on cytotoxic activities of the LGL population against all three target cell lines. Although both the PBL and LGL showed significant levels of CTX against K562, the PBL were not cytotoxic against the in vitro transformed autologous cell line VIP-F:T, while the LGL showed only marginal CTX against VIP-F:T. Neither the PBL nor LGL showed any CTX against the autologous normal fibroblast line Pen-F2. Treatment of the LGL with IFN- $\alpha$ or IL- 2 enhanced the cytotoxic activities of the LGL against K562 and VIP-F:T, but not against Pen-F2. In order to clearly rule out the possibility that the enhancement of NK activity seen with the partially purified IL-2 could be from undetectable amounts of interferon in the IL-2 preparation, experiments were performed using interferon-free IL-2 purified from conditioned media of the Gibbon leukemia cell line MLA-144, recombinant DNA-produced IL-2 (rIL-2), and recombinant interferon- $\gamma([$ rIFN- $\gamma]$ Genentech Inc., South San Francisco, CA). IL-2 purified from MLA-144 conditioned medium, rIL-2, and rIFN- $\gamma$, induced significant levels of cytotoxicity in the LGL against K562 and VIP-F:T (Fig. 2).

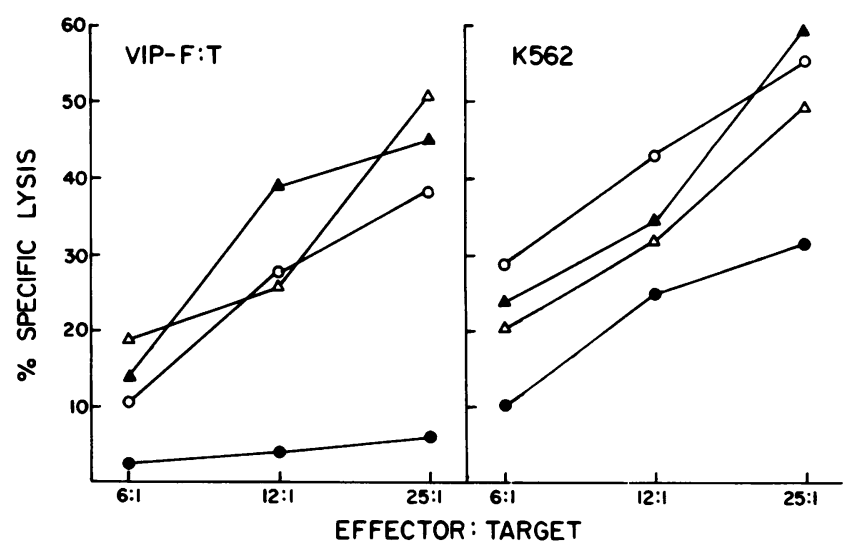

Figure 2. Cytotoxicity by LGL and the effect of interferon-free IL-2 and recombinant IFN- $\gamma$. LGL $\left(5 \times 10^{5} / \mathrm{ml}\right)$ were cultured overnight $(16 \mathrm{~h})$ with the following: $\bullet$, medium alone; $0,5 \times 10^{2} \mathrm{U} / \mathrm{ml}$ of rIFN- $\gamma ; \Delta, 5 \times 10^{2} \mathrm{U}$ of rIL-2; $\Delta, 5 \times 10^{2} \mathrm{U}$ of MLA-144 IL-2. 


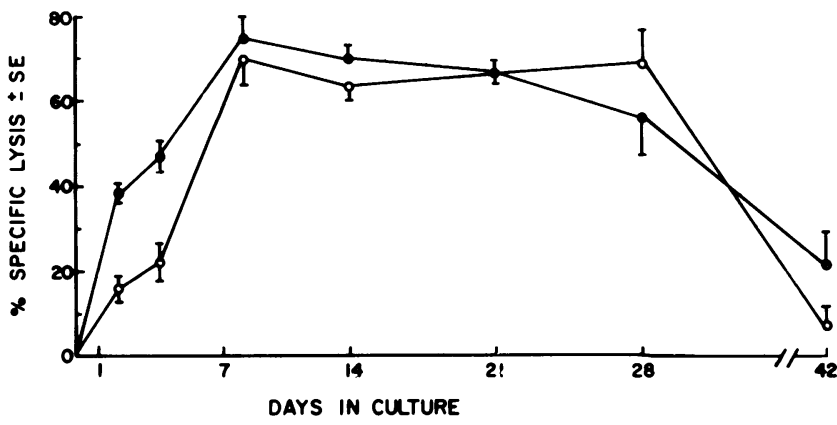

Figure 3. Kinetics of cytotoxic activity generated in IVC and IL-2 $(\bullet)$, or in IL-2 alone (0). (Effector:target ratio of 50:1. Differences in percentage specific lysis at 24 and $72 \mathrm{~h}$ were significant at $P<0.01$ or better by $t$ test.)

Although the cytotoxic activity by the PBL and the LGL against autologous VIP-F:T cells could be augmented by treatment with IFN- $\alpha$ or IL-2 for $2 \mathrm{~h}$ of incubation (data not shown), higher levels of activity were achieved after overnight incubation of the effector cells with either mediator. As shown in Fig. 3, high levels of CTX against VIP-F:T were generated following in vitro sensitization of the unfractionated effector cells with VIP-F:T cells in the presence of partially purified IL-2 or by culturing the effector cells in the presence of IL-2 alone. Although CTX of the effector cells reached peak levels by $7 \mathrm{~d}$, whether they were cultured in IL-2 alone or co-cultured with the VIP-F:T targets in the presence of IL-2, the levels of cytotoxicity generated by in vitro sensitization in IL-2 were significantly higher at 24 and $72 \mathrm{~h}$ of co-culture. CTX generated under either condition could be maintained for several weeks by culturing the effector cells in medium containing IL-2, after which activities generated under either condition declined (Fig. 3). Interestingly, when tested for natural killing against the autologous melanoma cells VIP, both PBL and LGL showed only marginal levels of CTX against the VIP cells (individual data not shown).

Phenotype of effector cell responding to IVC. The nature of the effector cells responding to IVC in the presence of IL-2 was examined by sorting effector cells by negative and positive selection in a FACS using monoclonal Leu-7 (HNK-1), Leu11a, OKT3, OKT4, or OKT8 antisera. Fig. 4 shows examples of some populations of cells that were sorted $1 \mathrm{w}$ after IVC. The cytotoxic activity was restricted to a population of cells bearing the Leu-7 and Leu-1 la phenotypes (Fig. 5). As shown in Fig. $5 A$, the removal of the Leu-7+ and Leu-11a+ cells eliminated the CTX almost completely, while the removal of the T4 and T8+ cells enhanced the level of CTX by enriching the NK cells in the effector population. Interestingly, the positively sorted Leu-7+ and Leu-11a+ cells immediately after sorting exhibited activity levels considerably lower than that of the unsorted population (Fig. $5 \mathrm{~B}$ ); this was possibly due to modulation of the respective molecule from the cell surface. After overnight culture, the Leu-7 and Leu-11a+ fractions showed higher levels of killing (Fig. $5 C$ ).

Several other sorting experiments were performed at different kinetic points of the IVC protocol. Abrogation of
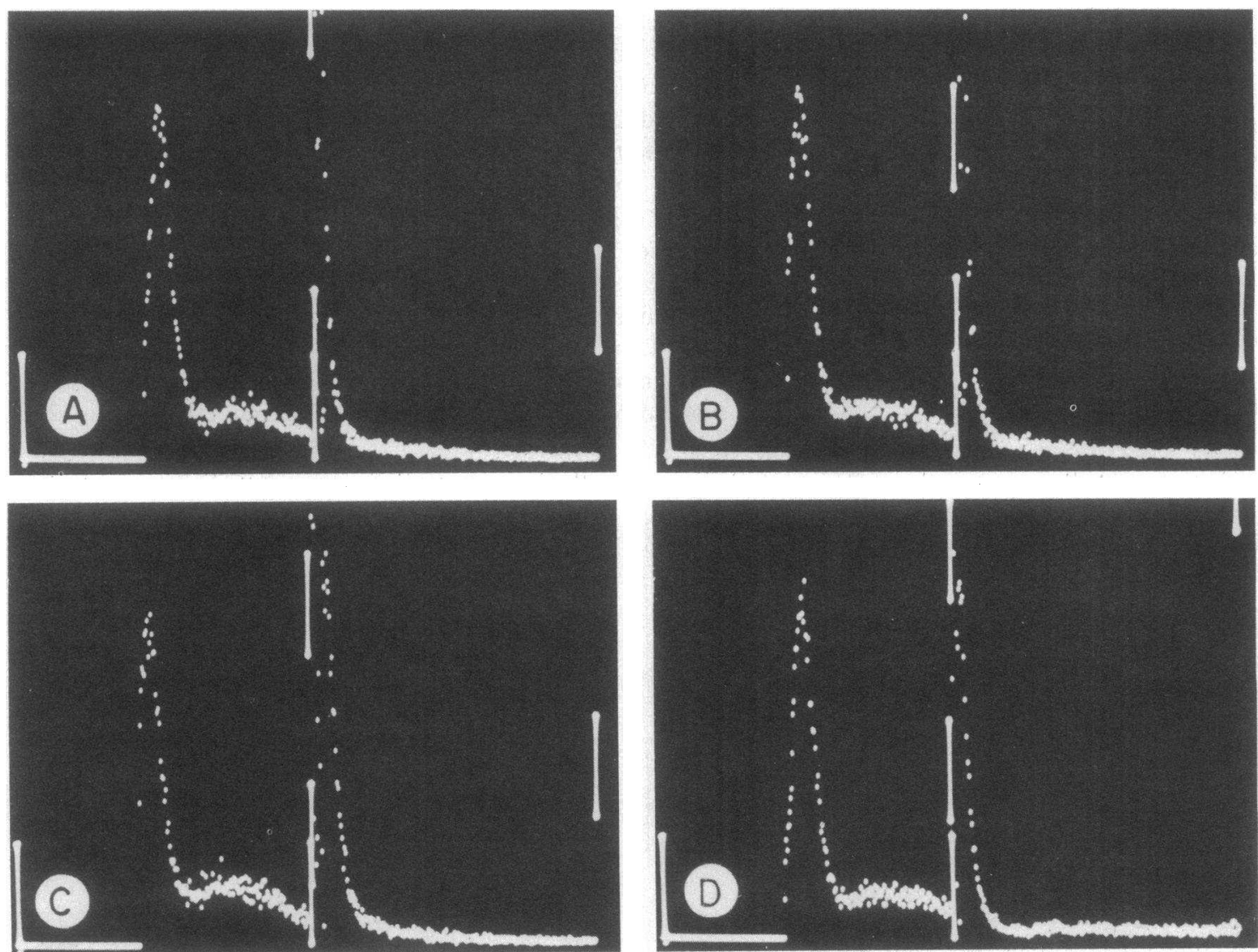

Figure 4. Fluorescence-activated cell sorting of the cytotoxic effector cells generated in co-culture for 1 wk with VIP-F:T in the presence of IL-2. ( $A$, Leu 7 [12.8\%] sort; $B$, Leu 11a [11\%] sort; $C$, T4 [22\%] sort; and $D$, T8 [31\%] sort.) 


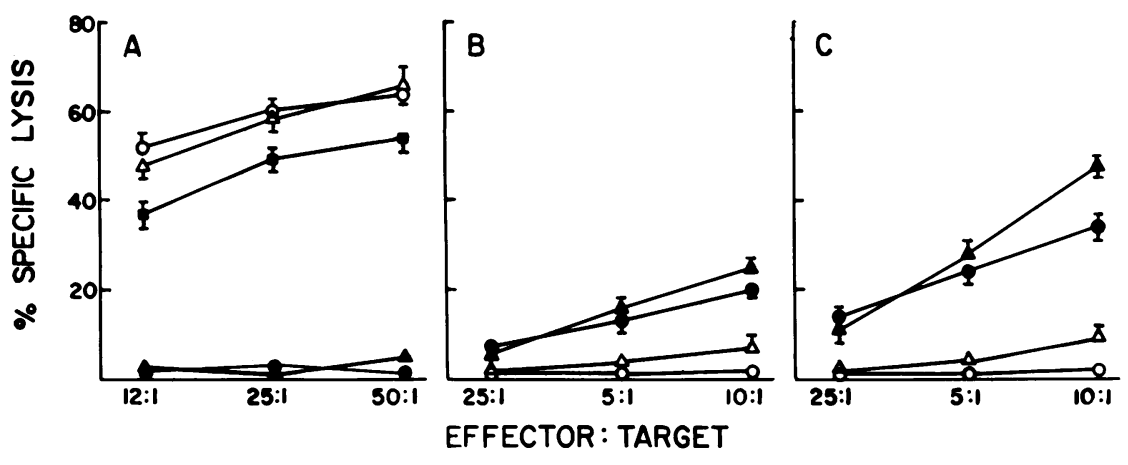

Figure 5. Fluorescence-activated cell sorting analysis of the phenotype of effector cells generated in co-culture for $7 \mathrm{~d}$ with VIP-F:T in the presence of IL-2. ( $A$ : $\mathbf{n}$, unsorted; O, T4population; $\triangle$, T8- population; $\bullet$, Leu 7population; and $\Delta$, Leu $11 \mathrm{a}-$ population.) $(B$ : •, Leu 7+ population; $\Delta$, Leu $11 \mathrm{a}+$ population; $\mathrm{O}, \mathrm{T} 4+$ population; and $\Delta, \mathrm{T} 8+$ population, assayed immediately after sorting.) ( $C$ : Same (as in Fig. 5 B) populations of antigen plus populations assayed for cytotoxicity after overnight culture. cytotoxic activity was reproducibly associated with the removal of the Leu-7+ or Leu-11a+ cells, not with the removal of OKT $8+$ or OKT3+ cells. Table I shows the results of several sorting experiments using different antisera at several different kinetic points of IVC. The activity, again, could be abrogated almost totally with the removal of the Leu-7+ or Leu-11a+ population.

Phenotypic characteristics of effector cell populations responding to several other autologous tumor lines (derived from surgical specimens including the melanoma line, VIP, derived from the same patient from whom the in vitro transformed line VIP-F:T was established) were examined for comparison. Table II shows the results of experiments in three separate systems. As can be seen, in vitro co-culture in IL-2 of autologous PBL generated cytotoxic populations bearing OKT8+ as well as Leu-7 phenotype. Interestingly, in all of these systems, PBL populations showed either marginal levels of, or no demonstrable level of, cytotoxicity before IVC (individual data not shown).

Specificities of cytotoxicity by the effector cells activated in IVC protocol. The specificities of the cytotoxic activities generated by in vitro sensitization in the presence of IL-2, or generated in IL-2 alone, were examined in cold target competition experiments (9). Interestingly, IVC against VIP-F:T in the presence of IL-2 generated a population of effector cells that showed restricted specificity when tested against VIP-F:T (Fig. 6). Neither the autologous nontransformed fibroblasts nor the NK prototype target K562 effectively competed against the VIP-F:T target. When the same effector cells were tested against K562, no restricted specificity was seen (Fig. 6). The

Table I. Cytotoxicity by Various Effector Cell Fractions at Different Kinetic Points of the IVC

\begin{tabular}{llll}
\hline & \multicolumn{3}{l}{$\begin{array}{l}\text { Percentage specific lysis against } \\
\text { VIP-F:T at E:T }=50: 1\end{array}$} \\
\cline { 2 - 4 } & $1 \mathrm{wk}^{*}$ & $3 \mathrm{wk}^{*}$ & $6 \mathrm{wk}^{*}$ \\
\hline Unsorted & 49 & 59 & 20 \\
T8 $^{-}$ & $63(7) \ddagger$ & $\mathrm{NT}$ & $34-(\mathrm{NT})$ \\
Leu 7- & $2(23)$ & $\mathrm{NT}$ & $3-(12)$ \\
Leu $11 \mathrm{a}^{-}$ & $4(20)$ & $7.8(12)$ & $\mathrm{NT}$ \\
T4 $^{-}$ & $63(0)$ & $\mathrm{NT}$ & $\mathrm{NT}$ \\
$\mathrm{T}^{-}$ & $\mathrm{NT}$ & $71(5.9)$ & $\mathrm{NT}$
\end{tabular}

* Experiments performed at 1,3 , or $6 \mathrm{wk}$ after initiation of IVC. $\ddagger$ The figures in parentheses represent percentage of specific lysis by the respective antigen-positive fractions tested immediately after sorting at E:T $=10: 1$. NT, not tested; E:T, effector:target. cytotoxic activities generated in IL-2 alone did not show restricted specificity when tested against VIP-F:T and K562 in reciprocal experiments (Fig. 6, $C$ and $D$ ). Specificity analysis was further extended using PBL sensitized in similar IVC protocol against the autologous nontransformed fibroblasts and against the NK prototype target K562. No cytotoxic activities exhibiting specificity against the VIP-F:T target demonstrable in cold target interaction experiments were observed (Fig. 7). Interestingly, in the other three autologous systems (in which data on phenotypic analysis of the IVC activated effector populations had been shown earlier in Table II), no target receptor specificity for the autologous tumor cells could be demonstrated at population levels by cold target competition assays (individual data not shown).

Confirmation of phenotypic analysis and target receptor specificity by positive selection of NK cells. Leu-11a+ cells, comprising $24 \%$ of the effector cell population generated in IVC at $2 \mathrm{wk}$, were sorted by positive selection under sterile conditions. The positively sorted Leu-1 1a + cells were cultured for $48 \mathrm{~h}$ in $20 \%$ IL-2 after which they were tested for cytotoxicity and specificity for target receptors. After $48 \mathrm{~h}$

Table II. Phenotypic Analysis of Effector Cell Populations Activated in Autologous Co-Culture in IL-2*

\begin{tabular}{lrrr}
\hline & \multicolumn{3}{c}{$\%$} \\
\cline { 2 - 3 } \%ffectors & $12: 1$ & $25: 1$ & $50: 1$ \\
\hline Case 1: VIP & & & \\
Unsorted & 22 & 29 & 41 \\
OKT8 $^{-}$ & 1 & 4 & 6 \\
HNK-1 & 12 & 15 & 28 \\
Case 2: PJ & & & \\
Unsorted & 17 & 28 & 38 \\
OKT8- & 5 & 14 & 18 \\
HNK-1 & 10 & 19 & 27 \\
Case 3: JH & & & \\
Experiment 1 & & & \\
Unsorted & 5 & 9 & 17 \\
HNK-1 & 3 & 7 & 9 \\
Experiment 2 & & & \\
Unsorted & 17 & 26 & 36 \\
OKT8- & 0 & 2 & 5 \\
\hline
\end{tabular}

* All sorting experiments were performed between $10 \mathrm{~d}$ and $3 \mathrm{wk}$ after IVC. Phenotypic analyses by cell sorting in the JH system were performed on two separate days 1 wk apart. 

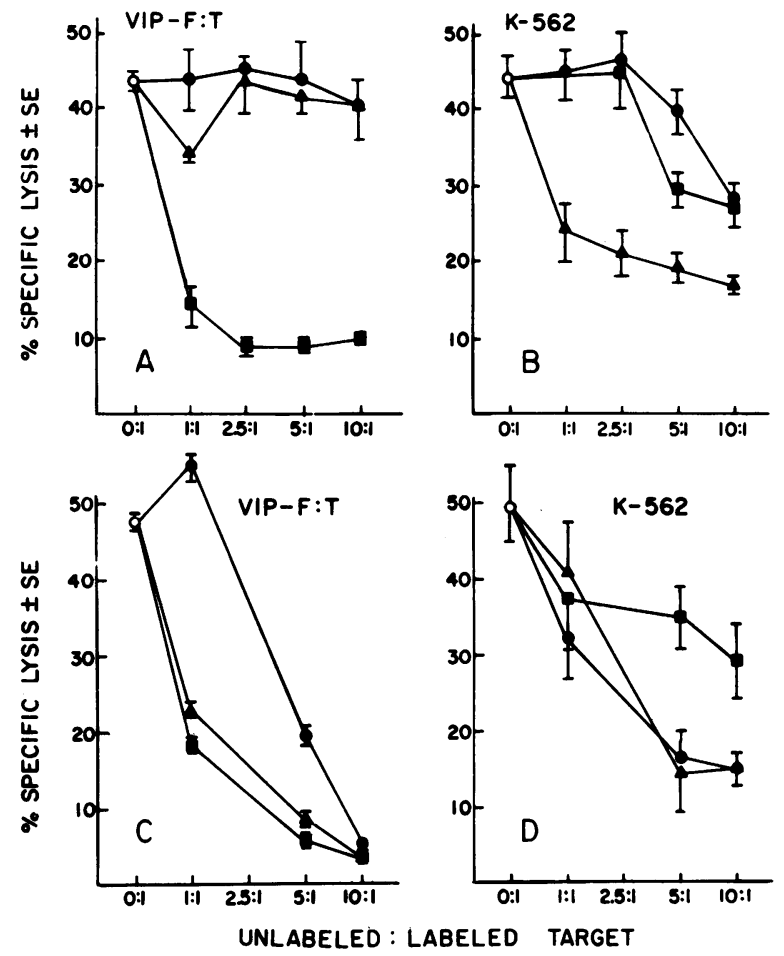

Figure 6. Analysis of specificity of cytotoxic activities in cold target competition assay. Effector cells generated in co-culture with VIP-F:T in the presence of IL-2 were tested against VIP-F:T ( $A$, at effector: labeled VIP-F:T target of 50:1) and against K562 (B, at effector: labeled K562 of 50:1) in the presence of unlabeled VIP-F:T ( $\square$ ), unlabeled K562 cells ( $\triangle$ ), and unlabeled nontransformed fibroblasts Pen-F2 (๑). Effector cells generated in culture with IL-2 alone were tested against VIP-F:T ( $C$, at effector:labeled VIP-F:T of 50:1) and against K562 (D, at effector:labeled K562 of 10:1) in the presence of unlabeled targets as above. Spontaneous release of $51^{\mathrm{Cr}}$ from labeled VIP-F:T or K562 in the presence of the respective unlabeled targets without effector cells was within $1 \mathrm{SE}$ of the spontaneous release observed with medium alone.

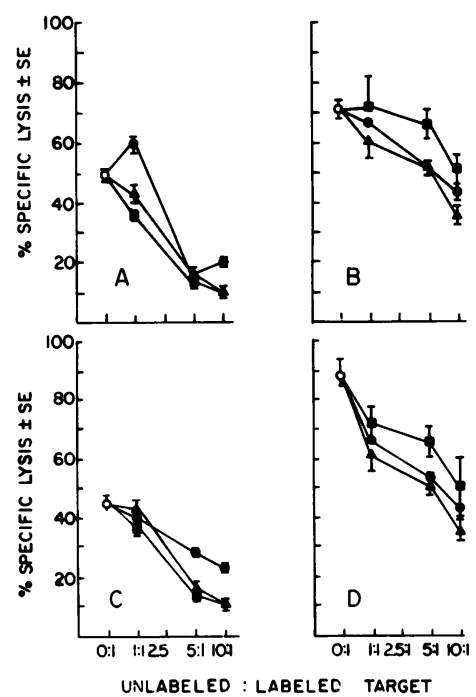

Figure 7. Analysis of specificity of cytotoxic activities in cold target competition assay. Effector cells generated in co-culture with Pen-F2 in the presence of IL-2 were tested against VIP-F:T $(A$, at effector:labeled VIP-F:T target of 50 : 1) and against $\mathrm{K} 562(B$, at effector:labeled $\mathrm{K} 562$ of 50 : 1) in the presence of unlabeled VIP-F:T ( () , unlabeled $\mathrm{K} 562$ cells $(\triangle)$, and unlabeled nontransformed fibroblasts Pen-F2 (๑). Effector cells generated in culture with $\mathrm{K} 562$ in the presence of IL-2 were tested against VIP-F:T $(C$, at effector:labeled VIP-F:T of 50:1) and against $\mathrm{K} 562$ (D, at effector: labeled K562 of 10:1) in the presence of unlabeled targets as above. Spontaneous release of ${ }^{51} \mathrm{Cr}$ from labeled VIP-F:T or K562 in the presence of the respective unlabeled targets without effector cells was within $1 \mathrm{SE}$ of the spontaneous release observed with medium alone.
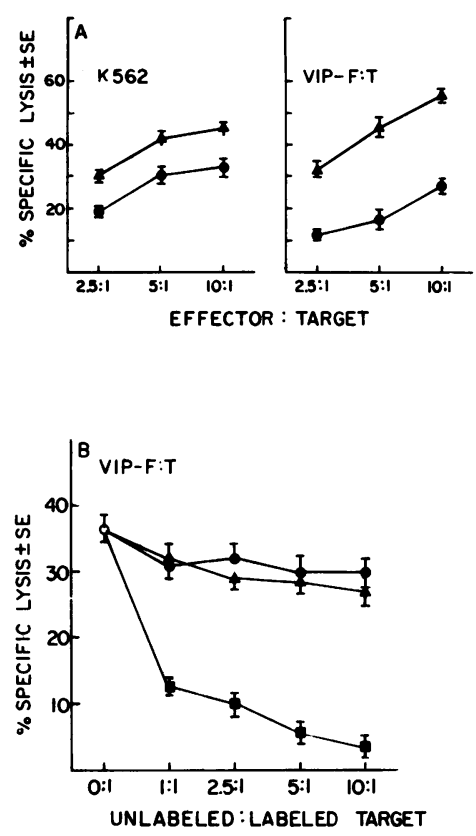

Figure 8. (A) Phenotypic analysis of cytotoxic effector cells generated in coculture for 2 wk with VIP. $\mathrm{F}: \mathrm{T}$ in the presence of IL-2 by positive sorting. Unsorted effectors $(\bullet)$, Leu$11 \mathrm{a}+$ population $48 \mathrm{~h}$ after sorting (A). (B) Analysis of specificity of cytotoxicity by the positively sorted Leu11 a + effectors in cold target competition assay. Leu$11 \mathrm{a}+$ effectors were tested against the VIP-F:T target at ratios of $5: 1$ in the absence of unlabeled targets (o); they were tested in the presence of unlabeled VIP. F:T (๘), unlabeled K562 $(\Lambda)$, and unlabeled Pen-F2 (๑).

culture in $20 \%$ IL-2, the positively sorted Leu-11a+ cells showed significant levels of cytotoxicities (Fig. $8 \mathrm{~A}$ ), as well as target receptor specificity for the autologous target VIP-F:T (Fig. 8 B).

In vivo protection by IVC effector cells against tumorigenic challenge by VIP-F:T target. The results of an in vivo tumor cell neutralization experiment is shown in Table III. As can be seen, the effector cells generated in IVC protocol clearly demonstrated protection of the nude mice from tumorigenic challenge with the VIP-F:T.3 cells. The cloned line was used in the transplantation experiment to avoid the potential problem of heterogeneity within the parent line. The cloned target cells were quite sensitive to lysis by the effector cells in vitro (data not shown). Table IV shows the effect of removal of the Leu$7+$ cells on the protective effect of the in vitro sensitized effector cells. In order to assure absolute sterility, the Leu-7+ cells were removed by complement induced lysis of Leu-7 antibody coated cells. It is of interest that removal of the Leu$7+$ cells abrogated the protective ability of the co-culture activated effector cells against tumorigenic challenge, while the effector population, incubated with complement withour prior antibody incubation, showed protection at an effector to target ratio of 10:1.

Table III. In Vivo Tumor Cell Neutralization Assay

\begin{tabular}{lll}
\hline Injected with & $\begin{array}{l}\text { Number mice } \\
\text { injected }\end{array}$ & $\begin{array}{l}\text { Number showing } \\
\text { tumor growth* }\end{array}$ \\
\hline $\begin{array}{l}\text { VIP-F:T.3 alone } \\
\begin{array}{l}\text { VIP-F:T.3 plus sensitized } \\
\text { effectors }\end{array}\end{array}$ & 6 & 5 \\
& 6 & 0
\end{tabular}

* Mice injected with tumor cells alone developed tumors within 3-4 wk. One mouse died from hepatitis at 4 wk without evidence of tumor. All six mice injected with tumor cells and sensitized PBL have, thus far, been observed for $10 \mathrm{wk}$. The difference in the incidence of positive tumor taken between the two groups is significant at $P$ $<0.01$ by Chi Square analysis. 
Table IV. Effect of NK Cell Depletion

on In Vivo Tumor Cell Neutralization

\begin{tabular}{lll}
\hline Injected with & $\begin{array}{l}\text { Number mice } \\
\text { injected }\end{array}$ & $\begin{array}{l}\text { Number showing } \\
\text { tumor growth* }\end{array}$ \\
\hline $\begin{array}{l}\text { VIP-F:T.3 alone } \\
\begin{array}{l}\text { VIP-F:T.3 plus sensitized } \\
\text { effectors }\end{array}\end{array}$ & 6 & 6 \\
$\begin{array}{l}\text { VIP-F:T.3 plus Leu-7+ depleted } \\
\text { effectors }\end{array}$ & 6 & 0 \\
\hline
\end{tabular}

* All positive tumor growth was observed within $3 \mathrm{wk}$. Mice treated with sensitized effectors have been observed for tumor growth for 6 wk. The differences in the incidence of positive tumor taken between the treatment groups and the control group are significant at $P$ $<0.01$ by Chi Square analysis.

‡ For details of depletion technique, see Methods. $14 \%$ of the effectors stained positively in FACS with Leu-7 antiserum. After complement treatment of the Leu-7 antiserum, $16 \%$ of the population were found dead, while only $3 \%$ of the control population were lysed with complement treatment alone.

\section{Discussion}

This study represents an attempt to undertake a critical examination of natural immunity, from its theoretical point of view, against an in vitro transformed tumorigenic human cell line in an autologous situation, since the opportunity for such a study presented itself with the establishment of such a cell line. All experiments described in relation to the VIP-F:T system represent autologous reactions. Since no other in vitro transformed cell line and autologous blood lymphocytes were available to these investigators, comparitive experiments could be performed only in other autologous systems in which the tumor cell lines were derived from overt tumor specimens obtained from surgery. Admittedly, generalized conclusions regarding NK response against in vitro transformed tumorigenic cell lines cannot be made from observations made in one case study. Considering the unique nature of the observations and the interest in NK cell on immunosurveillance, however, the experiments on the VIP-F:T system described in this communication provide unique insights into and allow several conclusions regarding NK activity against an in vitro transformed tumorigenic autologous cell line. First, the existence of an interesting dichotomy in NK cell mediated immune response in this case is clearly demonstrable. While this individual could mount an NK response against the prototype target K-562, very little cytotoxic activity was found in the nascent state in either the PBL or their LGL fraction against the in vitro transformed autologous target VIP-F:T. Functional activation of the NK cells was required for cytotoxicity to be exhibited against the autologous target. Cytotoxic activation was achieved with certain IFN species and with IL-2. Second, the cytotoxic activity generated by IVC with the autologous VIP-F:T-associated antigen(s) in the presence of IL-2 resided almost exclusively in the population bearing OKT4 and OKT8 negative, Leu-7+, and Leu-11a+ phenotypes. Third, the autologous NK receptors for the in vitro transformed target VIPF:T was different from receptors for the prototype NK target K562. More important, the autologous effector activity gen- erated by IVC with the transformed target VIP-F:T in the presence of IL-2 clearly recognized the autologous transformed line from its normal nontransformed counterpart. No such target recognition was observed with the effector cells activated in IL-2 alone, or generated by IVC against the non-tumorigenic autologous fibroblasts. This suggests a specific receptor-mediated lytic interaction between activated cells and the in vitro transformed autologous cell line VIP-F:T.

Although autologous NK reactivities against other in vitro transformed tumorigenic cell lines could not be studied, the phenotypic characteristics and specificity analysis of cytotoxic reactivities generated in identical co-culture protocols in several autologous systems (in which the targets were derived from surgical specimens, including a melanoma line, VIP, from the same patient from whom the target line VIP-F:T was derived) differed remarkably from that observed in the VIP-F:T system. In none of these experiments involving co-culture against targets derived from in vivo grown tumors were the reactivities solely confined to the lineage bearing OKT8 negative, Leu-7+ populations; and no target receptor specificity could be demonstrated by cold target interaction technique at population levels. Although it is possible that the phenotypic characteristics, as well as the target receptor specificity of the IVC activated effector cells observed in the VIP-F:T system, might represent a unique phenomenon involving a single case, the possibility of the existence of different patterns of cytotoxic reactivities (observed in co-culture protocol described here) to overt tumor cells which presumably grow by escaping immunosurveillance and to in vitro transformed cells (which may not have been presented optimally to the immune system for the generation of cytotoxic $\mathrm{T}$ lymphocytes (CTL) response) cannot be ruled out.

It is not presently possible to offer a satisfactory explanation for why the IVC protocol, i.e., autologous co-culture against VIP-F:T in the presence of exogenous IL-2, did not generate a CTL response (cytotoxic effect bearing OKT4 or OKT8 phenotype). A possible explanation may lie in the fact that the autologous co-culture against the VIP-F:T target in the presence of IL-2 generated an effective, early NK response which in turn destroyed the antigen-bearing targets much too quickly to induce a classic CTL response. Indeed, regular inspections of the IVC culture wells indicated virtually total target cell destruction within 3-5 d.

It is tempting to call the precursors of the effector cells, activated in co-culture in the presence of IL-2 in the VIP-F:T system, NK cells simply because of phenotypic characteristics of the activated effectors. Although the phenotypic characteristics of the activated effector cells suggest that their precursors might have been NK cells or a lineage within the broad heterogeneous NK populations, the possibility cannot be ruled out that the activated effector cells represent an early $\mathrm{T}$ cell lineage without the differentiation antigens $\mathrm{T} 4$ or $\mathrm{T} 8$ and expressing the HNK-1 or Leu-11a phenotype. The specificity observed with these activated effector cells, however, represents a significant departure from the traditional notion of nonspecificity in natural immunity, and on this issue one might further question the NK terminology with reference to the precursors of the activated effector cells. In addition, the cytotoxicity by the effector cells activated in IVC in the presence of IL-2 may be construed as lymphokine activated killing (LAK), a phenomenon recently described in the literature 
(10). Admittedly, the question of target receptor specificity for NK cells has been elusive. A critical evaluation of specificities of NK cell mediated cytotoxicities has been hampered by a number of factors, two of which have been the lack of tumor models in which immune response by NK cells could be examined in its true, albeit theoretical, perspective and the extreme heterogeneity within the NK population. In this respect, this type of approach (i.e., studies of cytotoxic response to in vitro transformed autologous targets) offers a unique, if not ideal, opportunity to address these issues. In reference to LAK as a possible explanation of the activity inducible in IVC, two major points clearly suggest that the IVC protocol described in this communication generates a response which, by phenotypic and functional definition, is not an LAK response. LAK is mediated by a population bearing other than the Leu-7 phenotype; and LAK, as shown by Grim et al. (10) and confirmed in our studies (Fig. $6 \mathrm{C}$ ), shows no restricted specificity. The cytotoxic response generated in IVC in this study was clearly exhibited by a population bearing Leu-7 and Leu-11a phenotypes (by far the best phenotypic markers presently available for human NK cells); and such activity, at least by a population within the lineage broadly defined as NK, exhibited specificity for VIP-F:T target receptors distinguishable from that associated with the prototype NK target K562 or with the nontransformed autologous fibroblasts.

Finally, our data on transplantation rejection lend considerable credibility to the cytotoxicities observed in in vitro assays and confirm the in vitro observation of the effect of NK cell depletion on cytotoxicity. The data also point out that similar approaches employing in vitro transformed tumorigenic targets (a goal achievable by presently available technology, such as transfection and chemically induced transformation) offer opportunities to gain valuable insight into mechanisms of immunosurveillance against cancer.

\section{Acknowledgments}

This work was supported by grant CA 30461 from the National Cancer Institute.

\section{References}

1. Burnet, F. M. 1970. Immunological Surveillance. Pergamon Press, Ltd., Oxford. 280 pp.

2. Melief, C. J. M., and R. S. Schwartz. 1975. Immunocompetence and malignancy. In Cancer: A Comprehensive Treatise, Vol. 1. F. F. Becker, editor. Plenum Press, New York. 161-199.

3. Stutman, O. 1975. Immunodepression and malignancy. $A d v$. Cancer Res. 22:261-422.

4. Herberman, R. B., and J. R. Ortaldo. 1981. Natural killer cells: their role in defenses against disease. Science (Wash. DC). 214:24-30.

5. Mukherji, B., and T. J. MacAlister. 1983. Clonal analysis of cytotoxic T cell response against human melanoma. J. Exp. Med. 158: 240-245.

6. Mukherji, B., T. J. MacAlister, C. G. Gillis, D. C. Jeffers, and S. K. Slocum. 1984. Spontaneous in vitro transformation of human fibroblasts. J. Nat. Cancer Inst. 73:583-593.

7. Rabin, H., R. F. Hopkins III, F. W. Ruscetti, R. H. Neubauer, R. L. Brown, and T. G. Kawakami. 1981. Spontaneous release of a factor with properties of $T$ cell growth factor from a continuous line of primate tumor T cells. J. Immunol. 127:1852-1856.

8. Timonen, T., J. R. Ortaldo, and R. B. Herberman. 1981. Characteristics of human large granular lymphocytes and relationship to natural killer and K cells. J. Exp. Med. 153:569-582.

9. Herberman, R. B., M. E. Nunn, and H. T. Holden. 1976. Cytotoxicity inhibition assay for analysis of specificity of cell-mediated ${ }^{51} \mathrm{Cr}$ release cytotoxicity. In In Vitro Method in Cell-Mediated and Tumor Immunity. B. R. Bloom and J. R. David, editors. Academic Press, Inc., New York. 489-495.

10. Grimm, E. A., A. Mazumder, H. Z. Zhang, and S. A. Rosenberg. 1982. Lymphokine-activated killer cell phenomenon. $J$. Exp. Med. 155:1823-1841. 